\title{
Can Helicobacter pylori eradication affect long-term mortality?
}

\author{
Hang Lak Lee
}

Department of Internal Medicine, Hanyang University Seoul Hospital, Seoul, Korea

Received: April 10, 2021 Accepted: April 20, 2021

\author{
Correspondence to \\ Hang Lak Lee, M.D. \\ Department of Gastroenterology, \\ Hanyang University Seoul \\ Hospital, 222-1 Wangsimni-ro, \\ Seongdong-gu, Seoul 04763, \\ Korea \\ Tel: +82-2-2290-8354 \\ Fax: +82-2-2298-9183 \\ E-mail: alwayshang@hanyang.ac.kr \\ https://orcid.org/0000-0002- \\ 2825-3216
}

\section{See Article on Page 584-595}

Gastric cancer is the one of the most common cancers in Korea. From a global perspective, gastric cancer ranks third in cancer-related mortality [1]. Helicobacter pylori was classified as a Group 1 carcinogen for gastric cancer by the International Agency for Research on Cancer [2]. Therefore, there have been many studies on stomach cancer reduction after H. pylori eradication, particularly as to whether $H$. pylori eradication treatment could have a significant effect on the group at high risk for stomach cancer. Patients with early gastric cancer who received $H$. pylori treatment had lower rates of metachronous gastric cancer [3]. A recent meta-analysis of randomized controlled trials conducted in asymptomatic general populations reported a $34 \%$ reduction in the incidence of gastric cancer after $\mathrm{H}$. pylori eradication [4]. Overall, we believe that eradication of $\mathrm{H}$. pylori can lower the incidence of gastric cancer and may also reduce all-cause mortality. However, interestingly, one meta-analysis also demonstrated a non-significant increased risk in overall mortality with H. pylori treatment (risk ratio, 1.09; 95\% confidence interval [CI], 0.86 to 1.38 ) [5]. In addition, another study demonstrated a reduced risk of gastric cancer but, again, showed a nonsignificant increased risk of all-cause mortality among patients randomized to eradication therapy compared to patients in a control group (hazard ratio, 1.95; 95\% CI, 0.72 to $5.27 ; p=0.19$ ) [3]. These data suggest a potential harmful effect of $H$. pylori treatment in terms of increased non-gastric cancer mortality. This is a concern for a doctor prescribing eradication medicine and may damage the legitimacy of $H$. pylori eradication. However, the possible underlying mechanisms remain unclear, and this is not established theory. In Korea, gastric cancer was the most commonly diagnosed cancer in 2015, and the estimated prevalence of $H$. pylori infection was approximately $54 \%$ [6]. Therefore, unlike some other countries, this issue is of great significance in Korea.

In this issue of the Korean Journal of Internal Medicine, Kim et al. [7] reported their study "Effect of Helicobacter pylori treatment on the long-term mortality in patients with type 2 diabetes." This was a population-based retrospective cohort study using the Korean National Health Insurance Service-National Sample Cohort database and showed contradictory results compared to a previous meta-analysis in type 2 diabetes patients. Type 2 diabetes is an important risk factor for cardiovascular disease, cerebrovascular disease, and cancer $[8,9]$. The study of Kim et al. [7] showed that long-term overall 
mortality risk was not increased after $H$. pylori treatment in patients with type 2 diabetes. In addition, $H$. pylori treatment was not associated with an increase in mortality risk due to cardiovascular disease, cerebrovascular disease, and all cancers. During a median follow-up of 4.7 years, overall mortality was 5.9\% (101/1,727 patients) among patients in the $\mathrm{H}$. pylori-treatment cohort and 7.6\% (364/3,454 patients) among patients in the non-treatment cohort. In addition, the mortality risks due to cardiovascular disease (adjusted hazard ratio [aHR], 1.34; 95\% CI, 0.54 to 3.30; $p=0.529$ ), cerebrovascular disease (aHR, $0.97 ; 95 \% \mathrm{CI}, 0.37$ to $2.55 ; p=0.947$ ), and cancer (aHR, 1.08; 95\% CI, 0.68 to 1.72; $p=0.742$ ) were not significantly different between the groups. Despite several limitations as mentioned in the article, this study gives us valuable information about the mortality risks due to cardiovascular disease, cerebrovascular disease, and overall cancers after $H$. pylori eradication. This has great significance in that the study was conducted in diabetic patients, who are a high risk group for cardiovascular disease.

In conclusion, although this had the limitation of being a retrospective study, $H$. pylori eradication can be justified not only for gastric cancer patients but also for healthy $\mathrm{H}$. pylori carriers. A prospective long-term study should be performed to confirm this, and more research is needed to establish $\mathrm{H}$. pylori eradication treatment as the primary preventive treatment for gastric cancer in the general population.

\section{Conflict of interest}

No potential conflict of interest relevant to this article was reported.

\section{REFERENCES}

1. Bray F, Ferlay J, Soerjomataram I, Siegel RL, Torre LA, Jemal A. Global cancer statistics 2018: GLOBOCAN estimates of incidence and mortality worldwide for 36 cancers in 185 countries. CA Cancer J Clin 2018;68:394-424.

2. Schistosomes, liver flukes and Helicobacter pylori. IARC Working Group on the Evaluation of Carcinogenic Risks to Humans. Lyon, 7-14 June 1994. IARC Monogr Eval Carcinog Risks Hum 1994;61:1-241.

3. Choi IJ, Kook MC, Kim YI, et al. Helicobacter pylori therapy for the prevention of metachronous gastric cancer. $\mathrm{N}$ Engl J Med 2018;378:1085-1095.

4. World Health Organization. The top 10 causes of death [Internet]. Geneva (CH): World Health Organization, 2020 [cited 2021 Apr 21]. Available from: https://www.who.int/ news-room/fact-sheets/detail/the-top-10-causes-of-death.

5. Gyawali B, Kesselheim AS, D'Andrea E. Does Helicobacter pylori eradication therapy to prevent gastric cancer increase all-cause mortality? Int J Cancer 2019;144:411-412.

6. Hooi JKY, Lai WY, Ng WK, et al. Global prevalence of Helicobacter pylori Infection: systematic review and meta-analysis. Gastroenterology 2017;153:420-429.

7. Kim YI, Kim YA, Kim HJ, et al. Effect of Helicobacter pylori treatment on the long-term mortality in patients with type 2 diabetes. Korean J Intern Med 2021;36:584-595.

8. American Diabetes Association. 9. Cardiovascular disease and risk management: standards of medical care in diabetes-2018. Diabetes Care 2018;41(Suppl 1):S86-S104.

9. Giovannucci E, Harlan DM, Archer MC, et al. Diabetes and cancer: a consensus report. Diabetes Care 2010;33:16741685 . 